\title{
Inelastic Transport in Vibrating Disordered Carbon Nanotubes: Scattering Times and Temperature-Dependent Decoherence Effects
}

\author{
Hiroyuki Ishii, ${ }^{1,2}$ Stephan Roche, ${ }^{3,4}$ Nobuhiko Kobayashi, ${ }^{2}$ and Kenji Hirose ${ }^{5}$ \\ ${ }^{1}$ Department of Physics, University of Tokyo, 7-3-1 Hongo, Bunkyo-ku, Tokyo 113-0033, Japan \\ ${ }^{2}$ Institute of Applied Physics, University of Tsukuba, 1-1-1 Tennodai, Tsukuba, Ibaraki 305-8573, Japan \\ ${ }^{3}$ CIN2 (CSIC-ICN) Barcelona, Campus UAB, E-08193 Bellaterra, Spain \\ ${ }^{4}$ CEA, INAC, SP2M, L_Sim, 17 avenue des Martyrs, 38054 Grenoble, France \\ ${ }^{5}$ Nano Electronics Research Laboratories, NEC, 34 Miyukigaoka, Tsukuba, Ibaraki 305-8501, Japan
}

(Received 4 December 2009; published 19 March 2010)

\begin{abstract}
By using an order $N$ quantum transport methodology, and treating on the same footing static and dynamical disorders, we report on the theoretical exploration of quantum interferences tuned by electronphonon mediated decoherence mechanisms in disordered carbon nanotubes (with length up to $10 \mu \mathrm{m}$ ). This allows the extraction of inelastic scattering times together with temperature-dependent coherence lengths, which favorably compare with available experimental data at a quantitative level, and clarify the role of localization phenomena up to room temperature.
\end{abstract}

DOI: 10.1103/PhysRevLett.104.116801

PACS numbers: 73.63.Fg, 63.22.- m, 72.15.Lh

Introduction.-In recent years, the understanding of quantum transport properties of materials strongly modified by (electron-phonon mediated) time-dependent structural changes (such as DNA, organic crystals, etc.) has become a great theoretical challenge, genuinely demanding innovative computational approaches. Recent breakthroughs include the work of Troisi and co-workers [1], who performed nanoseconds-long molecular dynamics of a columnar mesophase of a discotic liquid crystal, from which realistic parameters of the Hamiltonian could be evaluated, allowing the exploration of electron dynamics in partially ordered organic semiconductors. Based on a similar strategy, Gutiérrez et al. [2] theoretically suggested that DNA conformational fluctuations are essential in determining long-range charge transport, shedding new light on long-standing controversies. In the same family of carbon based materials, carbon nanotubes (CNTs) [3] and graphene [4] are currently the subject of main scientific and technological concerns, but the role of temperaturedependent effects remains poorly understood. In a high bias regime, the true nature of inelastic transport through CNTs remains fiercely debated [5]. The situation is better understood at low bias, for which clean CNTs exhibit ballistic conduction up to the micron scale [6-8]. Weak localization (WL) $[9,10]$ or strong localization regimes [11] have also been reported in more disordered nanotubes, underlying the role of disorder-induced quantum interference effects up to room temperature. Finally, the temperature dependence of mean free paths (MFPs) was recently investigated experimentally in ultralong CNTs [12]. The importance of electron-phonon scattering processes was outlined, and an anomalous increase of resistance at low temperatures in very long CNTs was not understood.

In this Letter, by combining tight-binding molecular dynamics with an order $N$ real space method to compute the Kubo-Greenwood conductance, the temperature dependence of transport length scales in disordered CNTs is explored. Using the conventional phenomenology of WL theory, the coherence length limited by electron-phonon dephasing mechanisms is evaluated and compared with the inelastic scattering lengths derived from the lattice vibration properties. The crossover from a coherent to a fully incoherent transport regime is pinpointed by comparing such transport length scales, and is shown to be tuned by both static and dynamical disorders.

Decoherence mechanisms in mesoscopic systems take place as soon as dephasing processes start to reduce the quantum coherence of propagating wave packets. Depending on the temperature range and material under study, decoherence mechanisms can be driven either by electron-electron $(e-e)$ or electron-phonon (e-ph) couplings $[13,14]$. Within the WL theory, the quantum correction to conductance is related to the coherence length $L_{\phi}$, which denotes the scale beyond which the contribution of quantum interferences vanish. Here, the possible $e-e$ decoherence mechanisms (predominant at very low temperatures) are disregarded.

Hamiltonian model.-The WL regime is induced by introducing a static source of elastic scattering, based on the commonly employed Anderson-type disorder potential [6]. The model consists in modifying the on site energies $\varepsilon_{i}$ of $\pi$ orbitals of a simple (nearest-neighbors) tight-binding Hamiltonian of a $(5,5)$ metallic armchair CNT [3]. The interval width $\left[-W \gamma^{0} / 2,+W \gamma^{0} / 2\right]\left(\gamma^{0}=2.5 \mathrm{eV}\right)$ in which $\varepsilon_{i}$ are randomly selected determines the disorder strength and the corresponding elastic MFP [7]. By setting $W=0.2$ in our model we can reproduce the typical elastic MFP of $400 \mathrm{~nm}$ also found in Ref. [7] close to the charge neutrality point. Beyond this range, strong quantum interferences lead to localization phenomena. 
The impact of lattice distortion generated by increasing the nanotube temperature is encoded in the off-diagonal coupling matrix elements of the Hamiltonian. The atomic displacements are generated using a tight-binding molecular dynamics scheme, which thus provides time- and temperature-dependent renormalized $\pi-\pi$ off-diagonal coupling elements $\gamma_{i j}(t)$, encompassing $e$-ph interaction within both harmonic and anharmonic regimes [15]. We employ the empirical form for energies of $\gamma_{i j}(t)=$ $\gamma_{i j}^{0}\left|\mathbf{R}_{i}^{0}-\mathbf{R}_{j}^{0}\right|^{2} /\left|\mathbf{R}_{i}(t)-\mathbf{R}_{j}(t)\right|^{2}$ [16] with $\gamma_{i j}^{0}=2.5 \mathrm{eV}$, where $\mathbf{R}_{i}(t)$ represents the atomic position at time $t$ and $\mathbf{R}_{i}^{0}$ is at equilibrium. The phonon-vibration effects are taken into account from the molecular dynamics (MD) simulation [7] using the Brenner-Tersoff potential for C-C bonds [17,18]. At fixed temperature $T$, the velocities of carbon atoms are normalized at each time step by the conditions of $\sum_{i=1}^{N_{c}} M_{c} \dot{\mathbf{R}}_{i}^{2} / 2=3 N_{c} k_{B} T / 2$, where $M_{c}$ and $N_{c}$ are mass and number of carbon atom, and $k_{B}$ is the Boltzmann constant.

Weak localization phenomenology.-The phasecoherence length $L_{\phi}$ is derived using the WL theory as follows. In the presence of static disorder, beyond the scale defined by the elastic MFP, quantum interferences produce a quantum correction to conductance which scales as $\delta G_{\mathrm{WL}}=-\left(2 e^{2} / h\right) L_{\phi} / L$ [13]. Here $L$ is the system length and $L_{\phi}$ is derived from the temperature-dependent dephasing mechanisms taking place in the material $[14,19]$. The resistance at zero temperature is thus entirely monitored by static impurity scattering and localization effects. Since the resistance is defined as the inverse of conductance $R=1 /\left(G_{\mathrm{imp}}+\delta G_{\mathrm{WL}}\right) \simeq 1 / G_{\mathrm{imp}}-(1 /$ $\left.G_{\text {imp }}\right)^{2} \delta G_{\mathrm{WL}} \equiv R_{\text {imp }}+\delta R_{\mathrm{WL}}$, the quantum correction $\delta R_{\mathrm{WL}}$ is given by $\delta R_{\mathrm{WL}} / R_{\mathrm{imp}}^{2}=\left(2 e^{2} / h\right)\left(L_{\phi} / L\right)$. Assuming the validity of the Matthiessen rule, the total resistance is given by $R(T)=R_{\mathrm{imp}}+R_{\mathrm{ph}}(T)+\delta R_{\mathrm{WL}}(T)$, from which the coherence length can thereby be deduced:

$$
L_{\phi}(T)=\frac{h}{2 e^{2}}\left\{\frac{\rho(T)-\rho_{\mathrm{imp}}-\rho_{\mathrm{ph}}(T)}{\rho_{\mathrm{imp}}^{2}}\right\},
$$

where $\rho(\equiv R / L)$ is the $1 \mathrm{D}$ resistivity in the diffusive regime.

To obtain $L_{\phi}$ from Eq. (1), we need $\rho(T), \rho_{\text {imp }}$, and $\rho_{\mathrm{ph}}(T) . \rho(T)$ and $\rho_{\mathrm{Ph}}(T)$ are obtained from $\rho(T)=$ $\left(h / 2 e^{2}\right)\left[v_{F} / 2 D_{\max }(W, T)\right] \quad$ and $\quad \rho_{\mathrm{ph}}(T)=\left(h / 2 e^{2}\right) \times$ $\left[v_{F} / 2 D_{\max }(W=0, T)\right]$. Here, $D_{\max }$ is the diffusion coefficient discussed later. Since $\rho(T \rightarrow 0)$ includes the WL effects, we extract $\rho_{\text {imp }}$ from the high-temperature limit as $\lim _{T \rightarrow+\infty}\left[\left\{\rho_{\text {imp }}+\rho_{\mathrm{ph}}(T)+\delta \rho_{\mathrm{WL}}(T)\right\}-\rho_{\mathrm{ph}}(T)\right]$, assuming that the localization effect vanishes in the hightemperature limit, i.e., $\delta \rho_{\mathrm{WL}}(T \rightarrow+\infty)=0$.

Transport methodology.-To evaluate $L_{\phi}$ using Eq. (1), we employ the quantum wave packet approach [7], suitable to simulate quantum transport in huge and complex sys- tems. The resistivity $\rho(T)$ is obtained from the Kubo formula, such as $\rho(T)=\left(1 / 2 e^{2}\right) \operatorname{Tr}[L / \delta(E-\hat{H})] \times$ $\left(1 / D_{\max }\right)$. Here $\operatorname{Tr}[\delta(E-\hat{H}) / L]$ corresponds to the density of states per unit length at Fermi energy $E$, and $D_{\max }$ is the quantum diffusion coefficient given as the maximum value of the time-dependent diffusion coefficient, defined as $D(t) \equiv \int_{-t}^{+t} d t\left\langle\hat{v}_{x}(t) \hat{v}_{x}(0)\right\rangle_{E}$, which also reads

$$
D(t)=\frac{1}{t} \frac{\sum_{n}\left\langle\Psi_{n}\left|\delta(E-\hat{H})\left(\hat{U}^{\dagger}(t) \hat{x} \hat{U}(t)-\hat{x}\right)\right|^{2} \Psi_{n}\right\rangle}{\sum_{m}\left\langle\Psi_{m}|\delta(E-\hat{H})| \Psi_{m}\right\rangle},
$$

where $\hat{v}_{x}$ and $\hat{x}$ are the velocity and position operator along the tube axis in the Schrödinger representation, respectively. To evaluate $D(t)$, the dynamics of electronic wave packets $\left|\Psi_{n}\right\rangle$ is followed through the time-evolution operator $\hat{U}(t)=\Pi_{n=0}^{N-1} \exp [i \hat{H}(n \Delta t) \Delta t / \hbar]$, with $\hat{H}(t)$ and $\Delta t=t / N$. Employing the Chebyshev polynomial expansion method for $\hat{U}(t)$, an order $N$ calculation allows us to perform quantum transport calculations in systems with $\sim 10^{6}$ of carbon atoms [7]. Two initial electron wave packets are used, as well as eight distributions for the Anderson impurities, and eight initial Maxwell velocity distributions in the MD simulation, thereby averaging totally by $2 \times 8 \times 8$ different initial conditions. The calculations are performed for $D(t)$ up to $t \approx 10$ ps with $\Delta t \equiv$ $0.1 \times h /(1 \mathrm{eV})=0.41 \mathrm{fs}$ for $10 \mu \mathrm{m}$-long $(5,5)$ CNTs with open boundary conditions, while the MD simulations are done for a $250 \mathrm{~nm}(5,5)$ CNTs with periodic boundary conditions, sufficiently large to achieve convergence of transport calculations [7].

Mean free paths.-Figure 1 shows the resistances of $(5,5)$ CNTs at $T=0$ and $60 \mathrm{~K}$ as a function of CNT length $L(W=0.2)$. First, the observed decay of the diffusion coefficient $D(t)$ at zero temperature (inset), driven by localization effects, is seen to be strongly reduced at $T=$ $60 \mathrm{~K}$. The length dependent resistance is further seen to

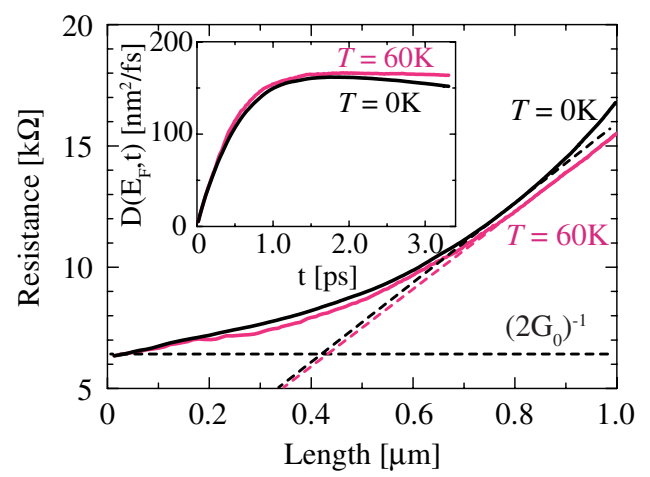

FIG. 1 (color online). Main frame: Length dependence of the total resistance for a $(5,5) \mathrm{CNTs}$ at $0 \mathrm{~K}$ (black line) and $60 \mathrm{~K}$ [gray (red) line] with $W=0.2$. Horizontal dashed line denotes $\left(2 G_{0}\right)^{-1}$, with $G_{0}=2 e^{2} / h$ the resistance quantum. The other dashed line is a guide to the eyes for pinpointing the crossover between ballistic and diffusive regimes. Inset: Time dependence of the diffusion coefficient for the same parameters. 
exhibit a crossover from a ballisticlike (almost length independent) to a diffusive behavior, in which the increase of resistance almost scales linearly with length. The crossing points of the two asymptotic lines for ballistic and diffusive regimes allow for an estimation of the MFP $\ell$, which is found as $\ell \approx 0.4 \mu \mathrm{m}$ at $T=60 \mathrm{~K}$ for $W=0.2$.

Now by tuning the static disorder parameter $W$, one is able to reproduce the experimental observations of the $T$ dependence of $\ell$. Since the velocity correlation vanishes with $\langle v(t) v(0)\rangle=\left\langle v_{F}^{2}\right\rangle \exp (-t / \tau)$, we obtain $D_{\max }$ as $D_{\max }=\left\langle v_{F}^{2}\right\rangle \tau$, and correspondingly MFP is obtained from $\ell=D_{\max } / v_{F}$. Here, $v_{F}$ is the Fermi velocity, which is evaluated as $0.82 \mathrm{~nm} / \mathrm{fs}$ from the simulation and perfectly agrees with the analytical estimate.

In Fig. 2, the logarithm plot of temperature-dependent $\ell$ is shown for $W=0,0.1$, and 0.2 . One clearly notices that MFPs are determined by the static disorder in low temperature regime $(T<50 \mathrm{~K})$, while they tend to merge in the high-temperature regime, when phonon scattering contributions to the transport properties prevail. For $W=0$, the MFP $\ell_{i e} \sim T^{-1}$ is fully dominated by phonon-driven scatterings [20]. More interestingly, the obtained temperature dependence of $\ell$ from our numerical results is in full agreement with experimental data [12]. We also find that the MFP increases linearly with CNT diameter (not shown here) as predicted analytically [6]. From here on, we fix the parameter $W=0.2$ and discuss the behavior of $L_{\phi}$.

Coherence length.-Since we employ the classical approach for the phonon vibrations using MD simulation, non-energy-conserving scattering processes are neglected, and only dephasing mechanisms driven by dynamical disorder are retained. This computational methodology can be rationalized following the picture of quantum decoherence as described by Stern et al. [19]. Dynamical disorder will generate many energy-conserving processes that yield a

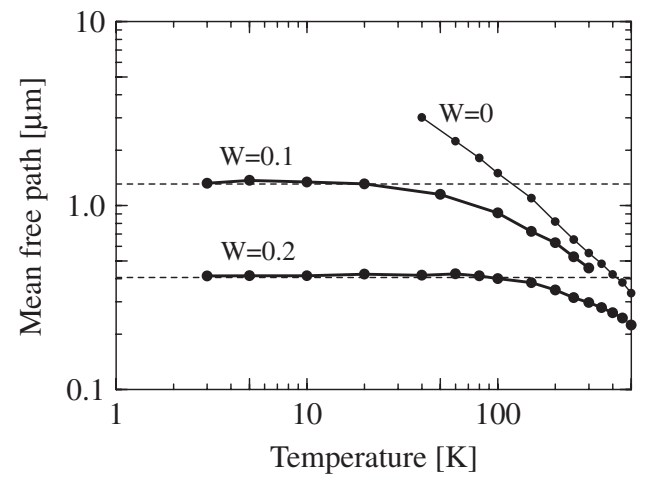

FIG. 2. Temperature dependence of the MFPs for a $(5,5)$ CNT with both dynamical disorder and several strengths of the static disorder potential $W$. For $W=0$, MFP is entirely determined by the $e$-ph coupling and is proportional to $T^{-1}$. For $W \neq 0$, MFP saturates to a $T$-independent value, tuned by the disorder strength. In high $T$ regime, MFP approaches the value in the $W=0$ limit, evidencing that phonon scatterings dominate the transport properties. dephasing of the propagating wave packets and corresponding loss of quantum coherence. This loss accumulates in time as the wave packet diffuses through the disordered system, and once the uncertainty on the quantum phase is in the order of $2 \pi$, the full suppression of coherence allows us to extract $L_{\phi}$ (see also [14]).

We evaluate the temperature dependence of resistivities from 3 to $550 \mathrm{~K}$, which are averaged within $[-0.25 \mathrm{eV},+0.25 \mathrm{eV}]$ energies. We find that $[\rho(T)-$ $\left.\rho_{\text {Ph }}(T)\right]$ saturates to $9.38 \times 10^{-3} \mathrm{k} \Omega / \mathrm{nm}$ over $400 \mathrm{~K}$, and the elastic MFP $\ell_{e}=688 \mathrm{~nm}$ since $\rho_{\text {imp }}=\left(h / 2 e^{2}\right) \times$ $\left(1 / 2 \ell_{e}\right)$. We can evaluate $L_{\phi}(T)$ from the obtained $\rho(T)$, $\rho_{\text {ph }}(T)$, and $\rho_{\text {imp }}$ using Eq. (1). Figure 3 gives the inverse phase-coherence length $L_{\phi}^{-1}$ as a function of $T$. We see that the temperature dependence of $L_{\phi}$ is markedly changed at $250 \mathrm{~K}$. The data from 20 to $100 \mathrm{~K}$ are fitted very well by $L_{\phi} \propto T^{-0.558}$, while $L_{\phi} \propto T^{-1}$ fits the data from 250 to $350 \mathrm{~K}$. This observation is well understood from the temperature dependence of relaxation times $\tau$. The inelastic relaxation time due to phonon scatterings is given by $\tau_{i e}(T) \equiv D_{\max }(W=0, T) / v_{F}^{2}$ and shows $T^{-1}$ dependence. On the other hand, the elastic relaxation time $\tau_{e}\left(\equiv \ell_{e} / v_{F}\right)$ due to impurity scatterings is temperature independent; thus, we see that $\tau_{i e}(T)$ and $\tau_{e}$ cross each other at $250 \mathrm{~K}$ (Fig. 3, inset). This indicates that, when $T$ is less than $250 \mathrm{~K}$, elastic scattering with impurities dominates and $e$-ph mediated scattering occurs after the system reaches the diffusive transport regime. In this regime, using the diffusion coefficient $D_{\max }^{e} \equiv v_{F} \ell_{e}$, the inelastic scattering length is determined by $\sqrt{D_{\max }^{e} \tau_{i e}}$, and therefore $L_{\phi}$ has the $T^{-0.5}$ dependence since $\tau_{i e}$ is proportional to $T^{-1}$. It should be noted that $L_{\phi}(T \rightarrow 0)$ has no meaning in the present calculation, since we treat the phonons semiclassically and since $e$ - $e$ scattering (which is important in such low temperature regime) is neglected.

In the high-temperature regime $(T \geq 250 \mathrm{~K})$, on the other hand, electron propagation is fully dominated by $e$-ph scatterings. The inelastic scattering length is given by $v_{F} \tau_{i e}$, which is proportional to the inverse temperature

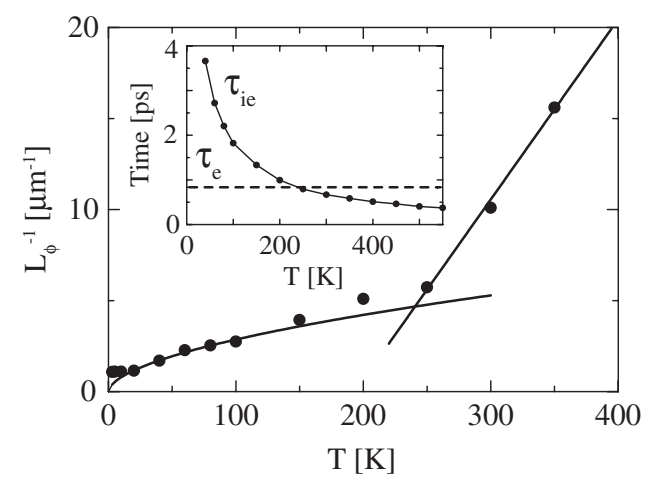

FIG. 3. Main frame: $L_{\phi}^{-1}$ vs $T$ curve for $(5,5) \mathrm{CNT}$ with $W=$ 0.2 . Inset: $T$ dependence of elastic and inelastic scattering times. The elastic scattering time equals the inelastic one at $250 \mathrm{~K}$. 


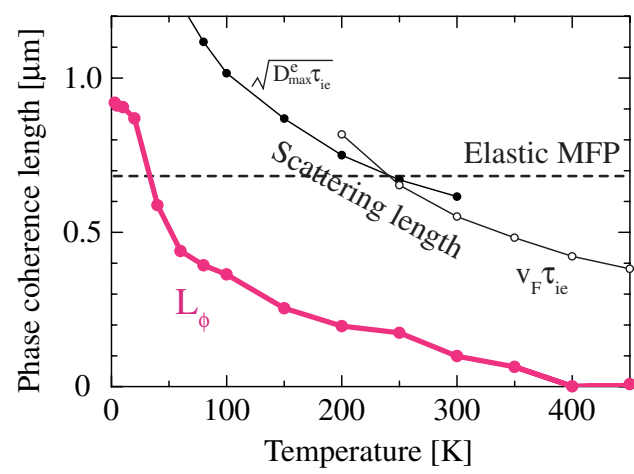

FIG. 4 (color online). Temperature dependence of phasecoherence length (thick red curve), together with the inelastic scattering lengths $\sqrt{D_{\max }^{e} \tau_{i e}}$ and $v_{F} \tau_{i e}$ (solid circles and open circles, respectively) and elastic MFP $\ell_{e}$ (horizontal dashed line).

$T^{-1}$. It is important to note that the coherence length is not determined from $L_{\phi} \sim \sqrt{D_{\max }^{e} \tau_{i e}}$ close to room temperature. These observations on the power-law behaviors of $T$-dependent inelastic scattering length agree qualitatively well with our simulation results of $L_{\phi}(T)$ in Fig. 3. The coherence length does not equal the inelastic scattering length because the quantum decoherence takes place before non-energy-conserving processes dominate conduction [19]. Here we note the $W$ dependence on $L_{\phi}(T)$. Since the elastic scattering time increases as $\tau_{e} \propto W^{-2}$, when the disorder $W$ decreases from 0.2 to 0.1 , the intersection of the temperature dependence of $\tau_{i e}(T)$ and $\tau_{e}$ is decreased from 250 to $50 \mathrm{~K}$. In such temperature range, $L_{\phi}$ shows $T^{-1}$ or $T^{-0.5}$ behavior depending on the static disorders of samples.

Stojetz and co-workers [10] reported on the energy dependence of both the elastic MFP $\ell_{e}$ and the coherence length $L_{\phi}$, particularly sensitive to onsets of new subbands. However, their experimental data also suggest that $L_{\phi} \leq$ $\ell_{e}$ close charge neutrality point, in apparent contradiction with the use of WL theory. Comparing $L_{\phi}$ with $\ell_{e}$ in Fig. 4, it is seen that for charge injection at low energies, WL theory is inapplicable whenever $T \geq 40 \mathrm{~K}$, which agrees with the inconsistency obtained in Ref. [10] at $T=60 \mathrm{~K}$. This underlines the strong decoherence effects driven by vibrations in CNTs, which also suggests some reconsideration of strong localization phenomena debated in the literature [11,12].

Conclusion.-Using a combined MD and order $N$ quantum transport methodology, the $e$-ph coupling effects on phase-coherence problems have been analyzed from lowto high-temperature regimes (but small bias voltage). Our simulations show that $L_{\phi}$ can reach $1 \mu \mathrm{m}$ at low temperatures (in metallic tubes), decays with temperature, and vanishes at $400 \mathrm{~K}$. At low temperatures, $L_{\phi}$ shows a $T^{-0.5}$ behavior satisfying the conventional WL theory, whereas $L_{\phi}$ decays linearly with temperature when inelas- tic scattering predominates over elastic processes. The crossover temperature $([50,250] \mathrm{K})$ depends on the strength of the static disorder. Finally, one notes that our results neglect the contribution of high-energy phonons, which more severely impact inelastic MFPs, but are only activated in the high bias regime [21].

This work was supported by the Next-Generation Super Computing Project, Nanoscience Program, and a Grant-inAid for Scientific Research from the MEXT of Japan. Calculations were performed at the Supercomputer Center, ISSP, University of Tokyo, and the Center for Computational Sciences, University of Tsukuba.

[1] A. Troisi, D. L. Cheung, and D. Andrienko, Phys. Rev. Lett. 102, 116602 (2009).

[2] R. Gutiérrez et al., Phys. Rev. Lett. 102, 208102 (2009).

[3] J. C. Charlier, X. Blase, and S. Roche, Rev. Mod. Phys. 79, 677 (2007).

[4] A. H. Castro Neto et al., Rev. Mod. Phys. 81, 109 (2009).

[5] V. Perebeinos, J. Tersoff, and Ph. Avouris, Phys. Rev. Lett. 94, 086802 (2005); M. Lazzeri et al., Phys. Rev. B 73, 155426 (2006); L.E. F. Foa-Torres and S. Roche, Phys. Rev. Lett. 97, 076804 (2006); P. Sundqvist et al., Nano Lett. 7, 2568 (2007).

[6] T. Ando, T. Nakanishi, and R. Saito, J. Phys. Soc. Jpn. 67, 2857 (1998); C. T. White and T. N. Todorov, Nature (London) 393, 240 (1998); P. L. McEuen et al., Phys. Rev. Lett. 83, 5098 (1999); F. Triozon et al., Phys. Rev. B 69, 121410 (2004).

[7] H. Ishii, N. Kobayashi, and K. Hirose, Appl. Phys. Express 1, 123002 (2008); H. Ishii et al., C.R. Physique 10, 283 (2009).

[8] D. Mann et al., Nano Lett. 3, 1541 (2003); A. Javey et al., Nature (London) 424, 654 (2003); A. Javey et al., Phys. Rev. Lett. 92, 106804 (2004).

[9] J. Appenzeller et al., Phys. Rev. B 64, 121404(R) (2001).

[10] B. Stojetz et al., Phys. Rev. Lett. 94, 186802 (2005).

[11] C. Gómez-Navarro et al., Nature Mater. 4, 534 (2005).

[12] M. S. Purewal et al., Phys. Rev. Lett. 98, 186808 (2007).

[13] E. Akkermans and G. Montambaux, Mesoscopic Physics of Electrons and Photons (Cambridge University Press, Cambridge, England, 2007).

[14] S. Chakraverty and A. Schmid, Phys. Rep. 140, 193 (1986).

[15] M. Gheorghe et al., Europhys. Lett. 71, 438 (2005); W. Rei et al., Phys. Rev. B 79, 161404 (2009).

[16] W. A. Harrison, Electronic Structure and the Properties of Solids: The Physics of the Chemical Bond (Dover, New York, 1989).

[17] W. C. Swope et al., J. Chem. Phys. 76, 637 (1982).

[18] D. W. Brenner, Phys. Rev. B 42, 9458 (1990).

[19] A. Stern, Y. Aharonov, and Y. Imry, Phys. Rev. A 41, 3436 (1990).

[20] H. Suzuura and T. Ando, Phys. Rev. B 65, 235412 (2002).

[21] J. Jiang et al., Phys. Rev. B 72, 235408 (2005); S. Roche et al., Phys. Rev. Lett. 95, 076803 (2005). 